\title{
Simulation modelling practices of ProSim03 participants: A survey
}

\author{
Rizwan Ahmed*, Tracy Hall*, Paul Wernick*, Stewart Robinson** \\ University of Hertfordshire, UK* \\ University of Warwick, UK** \\ R.2.Ahmed@herts.ac.uk*, T.Hall@herts.ac.uk*,P.D.Wernick@herts.ac.uk* \\ Stewart.Robinson@wbs.ac.uk**
}

"We need to get away from the crutch that modelling is an art."

(Gass 1987)

In this, paper we report the results of a preliminary survey of how people go about developing simulation models of software processes. The survey was conducted at ProSim03 Workshop. The main objective of this study is to identify current practices and issues in software process simulation modelling. We discuss the need for a formal process of software process simulation modelling. Moreover, we discuss potential critical issues in software process simulation modelling.

The Software process simulation modelling literature does not seem to report the process of simulation model development [6]. In most simulation studies of the software processes, modellers seem to be more interested in the end product rather than the process. This is analogous to the early days of software engineering when little attention was paid to the software development process.

Questions arise as to the process that simulation modellers do actually follow for model development. Eriksson [3] argues that a model's quality is questionable if it is developed using an ad-hoc process. Researchers in operations research (OR) suggest that in the absence of a systematic model development process a number of issues may arise such as: model quality, model confidence, lack of documentation, inadequate management and poor communication between stakeholders [8, 10]. Similar issues may also arise in software process simulation modelling. Many people consider that modelling is a creative and artistic activity and a formal process for model development is not really needed [10]. However, a formally established process has potential to help engineers produce high quality products with reduced time and cost [4]. This is because a formal process is more likely to bring discipline into human practices [9].

Simulation modelling of software processes has come into practice in the last decade. An extensive literature survey did not reveal any formal process in practice for software process simulation modeling. Kellner et al. [5] report a variety of issues, such as software process improvement, long-term software evolution modelling, control and operational management have been studied through software process simulation modelling. However, none of these studies seem to discuss the underlying process of model development. Rus et al. [6] appear to be the first to report the use of a formal process for simulation model development. Their proposed process has been derived from the collective experience of the authors. It is not based on a broader empirical assessment.

Given the lack of a model development process being reported in the software process simulation modelling literature, we analysed the Operation Research (OR) and Management Sciences (MS) literature. Pidd [7] says that modelling is the real technical heart of Operations Research and Management Sciences. Our analysis of the literature suggests that there is a growing feeling within operation research and management sciences that the modelling process needs to be more systematic. OR/MS researchers call for a discipline of "modelling science" [2]. Willemain [11] is a particular advocate of systemizing the model development process.

We report some results from the preliminary questionnaire survey of seventeen expert modelers in the field of software process simulation modelling. The objectives of this preliminary study are to:

1. Understand how modelers see themselves and their modelling practices

2. Establish if the modelling process is an issue amongst the software process simulation modelling community

3. Explore the most critical issues according to the software process simulation modelers' perceptions

The questionnaire consists of 6 sections, addressing the following questions:

1. Describe yourself as a modeler 
2. Describe the models you make

3. Describe the problems you model

4. Describe the most typical way you develop your models

5. Describe your modelling process

6. Describe the critical issues in software process simulation modelling.

The first four sections of the questionnaire replicate the questionnaire survey conducted by Willemain [11] with expert modelers in the field of operational research. We have added a fifth section to discover what process simulation modelers use for developing models of software processes and a sixth section to identify the most critical issues.

The sample for this survey is the participants of the ProSim03 workshop. Questionnaires were distributed to all 30 delegates and 17 responses were collected. To ensure the higher response rate the questionnaire was distributed and collected by hand. This helped avoiding the time and money costs of sending the questionnaire by post. A normal response rate is considered between $30-40 \%$ [1]. We obtained a better than average response rate of $56.67 \%$. This increases confidence in the study results.

Our empirical findings do not support our initial literature analysis that software process simulation modelling literature does not report use of a formal process for model development. However, majority of our sample reports that they use a systematic process for model development. The question is what kind of systematic process do they use? Most of the participants document their models formally. Though we do not yet know what formal documentation is to them and what the value of documentation is to model developers and model users. Most of the participants assess feasibility as a first step, define the problem sufficiently, value a modular structure, value usability, and review the model at each step. However, model evaluation is not much practiced. About half of the participants consider maintainability as an important aspect. Most of the participants show that they work on large and complex problems, and we would expect maintainability to be a potential issue for such large and complex models. Many participants do not have a life cycle perspective of the simulation modelling process. This may be a reason for not giving importance to maintainability. Majority of the participants consider modelling process and evaluation as the most urgent issues to be addressed in software process simulation modelling. Moreover, model design is considered to be the most urgent issue in the modelling process.

We were particularly interested in whether a formal modelling process is of interest to modelers or not. This initial research highlights the importance of a formal process for software process simulation model development. Furthermore, introduces many new questions that need attention from researchers in software process simulation modelling community.

\section{Reference:}

[1]. A.N. Oppenheim, "Questionnaire design, interviewing and attitude measurement". Continuum, London, (1992)

[2]. CONDOR, "Operations Research: The next decade", Operations Research, INFORM, (1988), Vol. 36, No. 4, pp619636

[3]. D.M. Eriksson, "A framework for the constitution of modeling processes: A proposition", European J. of OR, Elsevier Ltd, (2003), Vol. 145, pp.202-215

[4]. G. Cugola, and C. Ghezzi, "Software process: A retrospective and a path to the future", Software Process Improvement and Practice, John Wiley \& Sons Ltd., (1998), Vol. 4, pp. 101-123

[5]. I.M. Kellner, R. Madachy, and D. Raffo, "Software process simulation modeling: Why? What? How?" J. of Systems and Software, Elsevier Ltd, (1999), pp.91-105

[6]. I. Rus, H. Neu, and J. Munch,. "A systematic methodology for developing discrete event simulation models of software development processes", Proceedings of ProSim03 Workshop, Portland, Oregon, USA, (2003).

[7]. M. Pidd, "Just modeling through: A rough guide to modeling", INTERFACES, INFORMS, (1999), Vol. 29, No. 2, pp118-132

[8]. O. Balci, "Requirements for Model Development Environments", Computers and Operations Research, Elsevier Ltd, (1986), Vol. 13, pp.53-67.

[9]. P. Ferguson, W.S. Humphrey, S. Khajenoori, S. Macke, and A. Matvya, "Results of applying the Personal Software Process", Computer, IEEE, (1997), Vol. 30, No. 5, pp. 24 -31

[10]. S.I. Gass, "Managing the Modeling process: A personal reflection", European Journal of Operations Research, Elsevier Ltd, (1987) Vol. 31, pp1-8 
[11]. T.R. Willemain, "Insights on modeling from a dozen experts", Operations Research, INFORMS, (1994), Vol. 42, No. 2, pp213-222 